TRANSACTIONS OF THE

AMERICAN MATHEMATICAL SOCIETY

Volume 356, Number 8, Pages 3403-3404

S 0002-9947(04)03630-X

Article electronically published on March 30, 2004

\title{
ERRATA TO "ON THE STRUCTURE OF WEIGHT MODULES"
}

\author{
IVAN DIMITROV, OLIVIER MATHIEU, AND IVAN PENKOV
}

The main purpose of this errata list is to correct several inaccuracies and omissions that make it difficult to use the list in Section 7 of [1. We take this opportunity to also correct the typographical errors that we have noticed. The following inserts replace the corresponding original text. Negative line numbers indicate counting from the bottom of the page.

1. p. 2858 , line -1 :

"... is gratefully..."

2. p. 2860 , line -8 :

"... $M_{T}(\Omega)=\operatorname{ind}\left(\mathfrak{g}_{T}^{0} \oplus \mathfrak{g}_{T}^{+}, \mathfrak{g} ; \Omega\right), \ldots$ ".

3. p. 2866 , line 5 :

"and only finitely many non-isomorphic cuspidal $\mathfrak{g}_{0}$-submodules."

4. p. 2866, line 11:

"only have finitely many non-isomorphic irreducible $\mathfrak{g}_{0}$-submodules. ..."

5. p. 2866, line -18:

"(ii) Let $\mathfrak{s}$ be a good Levi subsuperalgebra ..."

6. p. 2867 , lines 11 through 16 :

"7.3. Let $\mathfrak{g}=\mathfrak{o s p}(n \mid 2 m)$. Then $\mathfrak{g}_{0} \simeq \mathfrak{o}(n) \oplus \mathfrak{s p}(2 m)$, and $\mathfrak{g}_{1} \simeq U \otimes V$ for $n \neq$ 2 , and $\mathfrak{g}_{1} \simeq V \oplus V^{*}$ for $n=2$, where $U$ and $V$ are respectively the natural representations of $\mathfrak{o}(n)$ and $\mathfrak{s p}(2 m)$. Any good Levi subsuperalgebra is of type $\mathfrak{o} \mathfrak{s p}\left(n^{\prime} \mid 2 m^{\prime}\right) \oplus \mathfrak{g l}(\mathbf{n}) \oplus \mathfrak{g l}(\mathbf{m})$, where $n^{\prime} \neq 2, n-n^{\prime}$ is even, and $\mathbf{n}$ and $\mathbf{m}$ are respectively partitions of $n-n^{\prime}$ and $m-m^{\prime}$. Such a subsuperalgebra is cuspidal iff $n^{\prime} \neq 2$ and $n^{\prime} \leq 6$. The latter follows from the fact that $\mathfrak{o}(k)$ is of type $A$ or $C$ for $k \leq 6$ and the fact that $\mathfrak{o s p}(2 \mid 2 m)$ has a $\mathbf{Z}$-grading $\mathfrak{g}^{-1} \oplus \mathfrak{g}^{0} \oplus \mathfrak{g}^{1}$ with $\mathfrak{g}^{0}=\mathfrak{g}_{0} . "$

7. p. 2867, line 24:

"... Any good Levi subsuperalgebra is cuspidal and ..."

8. p. 2867 , lines -11 through -8 :

"(iii) $\mathfrak{o s p}(1 \mid 2) \oplus \mathfrak{k}$, where $\mathfrak{k}$ is a Levi subalgebra of $\mathbf{G}_{2}$ which does not contain a short root of $\mathbf{G}_{2}$;

(iv) a $\mathfrak{g l}(1) \oplus \mathfrak{k}$, where $\mathfrak{k}$ is a proper Levi subalgebra of $\mathbf{G}_{2}$;

(v) $\mathfrak{o} \mathfrak{s p}(3 \mid 2) \oplus \mathfrak{g l}(1) . "$

9. p. 2867 , line -1 :

"(iii) $\mathfrak{o s p}(4 \mid 2) \oplus \mathfrak{g l}(1) ;$

10. p. 2868 , line 1 :

"(iv) a Levi subalgebra of $\mathfrak{g}_{0}$ which does not contain $\mathfrak{o}(7)$ and is not isomorphic to $\mathfrak{s l}(2) \oplus \mathfrak{g l}(2) \oplus \mathfrak{s l}(2) . "$

Received by the editors February 26, 2004.

2000 Mathematics Subject Classification. Primary 17B10. 
11. p. 2868, line 18:

" $m \neq n$, over $\mathfrak{p s l}(n \mid n)$, and over $\mathbf{W}(n)$. Indeed, if $\mathfrak{g}=\mathfrak{s l}(m \mid n), \mathfrak{g}=\mathfrak{p s l}(n \mid n)$, or $\mathfrak{g}=$ $\mathbf{W}(n), \ldots$.

12. p. 2868 , line 19 :

“... where $\mathfrak{g}^{0} \simeq \mathfrak{s l}(m, n)$ for $\mathfrak{g}=\mathfrak{s l}(m \mid n), \mathfrak{g}^{0} \simeq \mathfrak{s l}(n) \oplus \mathfrak{s l}(n)$ for $\mathfrak{g}=\mathfrak{p} \mathfrak{s l}(n \mid n)$, and"

13. p. 2868 , line 20 :

"... Moreover, the map $M \mapsto H^{0}\left(\mathfrak{g}^{-1}, M\right)$ is ..."

14. p. 2868, lines 23-24:

"... modules over $\mathbf{s p}(2 k+1)$ and $\mathbf{D}(2,1, \alpha) \ldots$...

15. p. 2868, lines 29-30:

“... modules over $\mathfrak{o} \mathfrak{s p}(n \mid m)$ (with $n \neq 2, n \leq 6), \mathfrak{p s q}(n)$, and $\mathbf{D}(2,1, \alpha)$. For the remaining ..."

\section{REFERENCES}

[1] Ivan Dimitrov, Olivier Mathieu and Ivan Penkov, On the structure of weight modules, Trans. Amer. Math. Soc. 352 (2000), 2857-2869. MR 2000j:17008

Department of Mathematics and Statistics, Queen's University, Kingston, Ontario, CANADA K7L 3N6

E-mail address: dimitrov@mast.queensu.ca

Université Louis Pasteur, IRMA, 7 rue René Descartes, 67000 Strasbourg, France

E-mail address: mathieu@math.u-strasbg.fr

Department of Mathematics, University of California at Riverside, Riverside, CalIFORNIA 92521

E-mail address: penkov@math.ucr.edu 